AEI-2000-067

OHSTPY-HEP-T-00-023

IFUM-FT-662

hep-th/0010137

\title{
Perturbative and instanton corrections to the OPE of CPOs in $\mathcal{N}=4 \mathrm{SYM}_{4}$
}

\author{
Gleb Arutyunov*,** 1, Sergey Frolov ${ }^{\ddagger, * *}{ }^{2}$ and Anastasios C. Petkou ${ }^{\dagger} 3$ \\ * Max-Planck-Institut für Gravitationsphysik, Albert-Einstein-Institut, \\ Am Mühlenberg 1, D-14476 Golm, Germany \\ $\ddagger$ Department of Physics, The Ohio State University \\ Columbus, OH 43210-1106, USA \\ †Dipartimento di Fisica dell'Universita di Milano, \\ Via Celoria 16, 20133 Milano, Italy
}

\begin{abstract}
We study perturbative and instanton corrections to the Operator Product Expansion of the lowest weight Chiral Primary Operators of $\mathcal{N}=4 \mathrm{SYM}_{4}$. We confirm the recently observed non-renormalization of various operators (notably of the double-trace operator with dimension 4 in the $\mathbf{2 0}$ irrep of $S U(4)$ ), that appear to be unprotected by unitarity restrictions. We demonstrate the splitting of the free-field theory stress tensor and R-symmetry current in supermultiplets acquiring different anomalous dimensions in perturbation theory and argue that certain double-trace operators also undergo a perturbative splitting into operators dual to string and two-particle gravity states respectively. The instanton contributions affect only those double-trace operators that acquire finite anomalous dimensions at strong coupling. For the leading operators of this kind, we show that the ratio of their anomalous dimensions at strong coupling to the anomalous dimensions due to instantons is the same number.
\end{abstract}

\footnotetext{
${ }^{1}$ email:agleb@aei-potsdam.mpg.de

${ }^{2}$ email:frolov@pacific.mps.ohio-state.edu

** On leave of absence from Steklov Mathematical Institute, Gubkin str.8, 117966, Moscow, Russia

${ }^{3}$ email:Anastasios.Petkou@mi.infn.it
} 


\section{Introduction}

$\mathcal{N}=4$ supersymmetric Yang-Mills theory $\left(\mathrm{SYM}_{4}\right)$ provides a concrete example of a supersymmetric quantum field theory where the idea of the AdS/CFT duality [1-3] can be successfully explored. According to the duality conjecture, $\mathrm{SYM}_{4}$ with a gauge group $S U(N)$ at large $N$ and at strong 't Hooft coupling $\lambda=g_{Y M}^{2} N$ is dual to type IIB supergravity on the $A d S_{5} \times S^{5}$ background. Unifying the results obtained in the context of the usual weak coupling expansion with the predictions of AdS/CFT duality allows us to conceive basic dynamical features of the theory.

In the superconformal phase the non-trivial dynamics of the $\mathrm{SYM}_{4}$ is encoded into correlation functions of gauge-invariant composite operators, which may acquire perturbative as well as non-perturbative (instanton) corrections. An important class of local operators in $\mathrm{SYM}_{4}$ is given by the Chiral Primary Operators (CPOs) of the form $O_{k}^{I}=\operatorname{tr}\left(\phi^{\left(i_{1}\right.} \ldots \phi^{\left.i_{k}\right)}\right)$, where $\phi^{i}$ are the Yang-Mills scalars. Under supersymmetry these operators generate short multiplets of the superconformal algebra $S U(2,2 \mid 4)$ that are dual to multiplets of type IIB supergravity compactified on $A d S_{5} \times S^{5}$. Unlike 2- and 3-point correlation functions of CPOs that are subject to the known non-renormalization theorems [4-12], 4-point functions receive in general perturbative and instanton corrections. As such, they contain important dynamical information for the supersymmetry multiplets which appear in the Operator Product Expansion (OPE) of two CPOs.

Recently, the 4-point function of the lowest weight CPOs $O_{2}^{I}$ has been computed in the supergravity approximation [13,14], and has been used in [15] to analyze their OPE at strong coupling. ${ }^{4}$ The structure of the OPE obtained in [15] for the first few low-dimensional operators was found to be in complete agreement with the predictions of AdS/CFT correspondence. Recall that the transformation properties of local gaugeinvariant operators of $\mathrm{SYM}_{4}$ with respect to the superconformal algebra allow one to classify them into three categories:

i) "Single-trace" chiral operators which belong to short representations and have conformal dimensions protected from quantum corrections.

ii) Operators which are obtained as "normal-ordered" products of the chiral operators. They may belong either to short or long representations, the former have protected conformal dimensions, while the dimensions of the latter are restricted from above.

iii) Operators which belong to long representations and whose conformal dimensions grow without bound in the strong coupling limit.

\footnotetext{
${ }^{4}$ Various aspects of 4-point functions involving operators descendent to $O_{2}^{I}$ were discussed in [16]-[30].
} 
According to the AdS/CFT duality, the operators in i) are dual to the type IIB supergravity fields while operators in ii) are dual to multi-particle supergravity states. For the operators in iii) the duality predicts the growth of their conformal dimensions as $\lambda^{1 / 4}$ when $\lambda \rightarrow \infty$. The latter operators are interpreted as being dual to string states (single- or multi-particle), which decouple in the strong coupling limit.

Comparison of the OPE of the two lowest weight CPOs in free-field theory and at strong coupling [15] has enabled us to make the following predictions for the structure of the OPE at finite $\lambda$ and $N$ :

i) The $R$-symmetry current and the stress tensor of the free-field theory, which involve only the six SYM scalars $\phi^{i}$, undergo splitting into 2 and 3 operators respectively belonging to different supermultiplets. Only one operator in each splitting is dual to a supergravity field and has protected conformal dimension, while all others decouple at strong coupling as their anomalous dimensions grow without bound.

ii) The only double-trace operator with free-field conformal dimension 4 that acquires an anomalous dimension at strong coupling is $O_{1}=: O^{I} O^{I}:+\cdots$ transforming in the trivial representation of the $R$-symmetry group. We argue that the free-field theory operator $O_{1}^{f r}$ also undergoes splitting into a sum of an operator dual to a gravity state and operators dual to string modes. The same kind of splitting also occurs for the scalar operator in the 84 irrep. The double-trace scalar operators in the 20 and 105 irreps do not split. The operator in the $\mathbf{2 0}$ irrep saturates the unitarity bound A') in the classification of [31] and is not protected from acquiring anomalous dimension. However, our analysis shows that this operator retains its canonical dimension and hence its non-renormalization is a genuine dynamical effect.

iii) The double-trace operator with free-field dimension 5 in the $\mathbf{1 5}$ irrep acquires anomalous dimension, while the one in the $\mathbf{1 7 5}$ has protected dimension. They both split at finite $\lambda$ and $N$.

iv) There are several towers of traceless symmetric tensor operators in the 105, 84 and 175 irreps, whose anomalous dimensions vanish.

Here we confirm the above predictions by analyzing the 4-point function of the CPOs $O_{2}^{I}$ computed at 2-loops in perturbation theory [32-35] (three-loop results were obtained in $[36,37])$. We also study the instanton contribution to the 4-point function. In [38] the correlation functions of the four $\mathcal{N}=2$ singlet scalar fields and of the sixteen dilatinos were computed in $\mathrm{SYM}_{4}$ with gauge group $S U(2)$ in the sector with instanton number $k=1$. In [39] these results were further generalized to the group $S U(N)$ and in [40,41] to arbitrary $k$ in the large $N$ limit. With the above results at hand, we then use the recently 
obtained non-renormalization theorem of [42] to restore the complete 4-point function of the CPOs $O_{2}^{I}$ and perform its OPE analysis. Our results are in agreement with the earlier considerations of [43] and show the absence of instanton contributions to the anomalous dimensions of single-trace operators in the Konishi multiplet [35]. Pictorially, we observe that the instanton contribution is "seen" only by those operators whose anomalous dimensions are non-zero and finite at strong coupling. In particular, the double-trace operator in 20 does not receive instanton corrections. Such a picture points to an interesting relation between the mysterious "multi-particle" supergravity states and the D-particle modes.

The plan of the paper is as follows. In Section 2 we recall the OPE structure of two CPOs at weak and strong coupling. In Section 3 we analyze the two-loop 4-point function of the lowest weight CPOs. We compute the anomalous dimensions of singleand double-trace operators and demonstrate the splitting of the free-field operators into distinct supermultiplets acquiring different anomalous dimensions. In Section 4 we study the instanton contribution to the 4-point function of the CPOs and show that instantons do not contribute to the anomalous dimensions of neither operators dual to string-modes nor operators with protected dimension. The dynamically protected operator in the $\mathbf{2 0}$ does not receive instanton contributions, which indicates that only operators receiving finite-anomalous dimensions at strong coupling "see" instantons. In the conclusion we discuss the results obtained.

\section{OPE algebra of CPOs at weak and strong coupling}

In this Section we review the structure of the OPE algebra of the lowest weight CPOs at both the weak and the strong coupling regimes. We follow the notation of [15] and also use

$$
\tilde{\lambda}=\frac{\lambda}{(2 \pi)^{2}}=\frac{g_{Y M}^{2} N}{(2 \pi)^{2}} .
$$

The normalized lowest weight CPOs in $\mathrm{SYM}_{4}$ are operators of the form

$$
O^{I}(x)=\frac{1}{2^{1 / 2} \tilde{\lambda}} C_{i j}^{I} \operatorname{tr}\left(: \phi^{i} \phi^{j}:\right)
$$

where the symmetric traceless tensors $C_{i j}^{I}, i, j=1,2, . ., 6$ form an orthonormal basis of the 20 of $S O(6)$. As was shown in [15] the leading terms in the OPE of two $O^{I} \mathrm{~s}$ in free field theory take the form

$$
O^{I_{1}}\left(x_{1}\right) O^{I_{2}}\left(x_{2}\right)=\frac{\delta^{I_{1} I_{2}}}{x_{12}^{4}}+\frac{2^{3 / 2}}{N} \frac{C^{I_{1} I_{2} I}}{x_{12}^{2}}\left[O^{I}\right]+\frac{2}{3^{1 / 2} N} \frac{\delta^{I_{1} I_{2}}}{x_{12}^{2}}[\mathcal{K}]
$$




$$
\begin{aligned}
& +\frac{2^{3 / 2}}{\tilde{\lambda} N} \frac{x_{12}^{\mu}}{x_{12}^{2}} C_{\mathcal{J}_{15}}^{I_{1} I_{2}}\left[J_{\mu}^{\mathcal{J}_{15}}\right]-\frac{\delta^{I_{1} I_{2}}}{6 \tilde{\lambda} N} \frac{x_{12}^{\mu} x_{12}^{\nu}}{x_{12}^{2}}\left[T_{\mu \nu}^{\text {free }}\right]+\frac{1}{\tilde{\lambda} N} \frac{x_{12}^{\mu} x_{12}^{\nu}}{x_{12}^{2}} C^{I_{1} I_{2} I}\left[T_{\mu \nu}^{I}\right] \\
& +\delta^{I_{1} I_{2}}\left[O_{1}\right]+C_{\mathcal{J}_{20}}^{I_{1} I_{2}}\left[O^{\mathcal{J}_{20}}\right]+C_{\mathcal{J}_{105}}^{I_{1} I_{2}}\left[O^{\mathcal{J}_{105}}\right]+C_{\mathcal{J}_{84}}^{I_{1} I_{2}}\left[O^{\mathcal{J}_{84}}\right] \\
& +C_{\mathcal{J}_{15}}^{I_{1} I_{2}} x_{12}^{\mu}\left[O_{\mu}^{\mathcal{J}_{15}}\right]+C_{\mathcal{J}_{175}}^{I_{1} I_{2}} x_{12}^{\mu}\left[O_{\mu}^{\mathcal{J}_{175}}\right]+\ldots
\end{aligned}
$$

Here $T_{\mu \nu}^{\text {free }}$ and $J_{\mu}^{\mathcal{J}_{15}}$ are respectively the stress tensor and the normalized $R$-symmetry current of the free field theory (including only six scalar fields), $\mathcal{K}$ is the normalized Konishi scalar, $T_{\mu \nu}^{I}$ is a traceless second rank tensor in 20 and $O^{\mathcal{J}}$ denote generically double-trace operators in the corresponding representation $\mathcal{J}$ of the R-symmetry. In addition to the above fields the OPE contains infinite towers of both single-trace as well as double-trace operators.

The strong coupling OPE compatible with the 4-point function of [13] is different from (2) and reads

$$
\begin{aligned}
O^{I_{1}}\left(x_{1}\right) O^{I_{2}}\left(x_{2}\right) & =\frac{\delta^{I_{1} I_{2}}}{x_{12}^{4}}+\frac{2^{3 / 2}}{N} \frac{C^{I_{1} I_{2} I}}{x_{12}^{2}}\left[O^{I}\right]+\frac{2^{3 / 2}}{3 \tilde{\lambda} N} \frac{x_{12}^{\mu}}{x_{12}^{2}} C_{\mathcal{J}_{15}}^{I_{1} I_{2}}\left[R_{\mu}^{\mathcal{J}_{15}}\right] \\
& -\frac{1}{30 \tilde{\lambda} N} \delta^{I_{1} I_{2}} \frac{x_{12}^{\mu} x_{12}^{\nu}}{x_{12}^{2}}\left[T_{\mu \nu}\right]+\delta^{I_{1} I_{2}} x_{12}^{\Delta_{1}^{(s)}}\left[O_{1}\right] \\
& +C_{\mathcal{J}_{20}}^{I_{1} I_{2}} x_{12}^{\Delta_{20}^{(s)}}\left[O^{\mathcal{J}_{20}}\right]+C_{\mathcal{J}_{105}}^{I_{1} I_{2}} x_{12}^{\Delta_{105}^{(s)}}\left[O^{\mathcal{J}_{105}}\right]+C_{\mathcal{J}_{84} I_{2}}^{I_{1} x_{12}} x_{84}^{(s)}\left[O^{\mathcal{J}_{84}}\right] \\
& +C_{\mathcal{J}_{15}}^{I_{1} I_{2}} x_{12}^{\Delta_{15}^{(s)}} x_{12}^{\mu}\left[O_{\mu}^{\mathcal{J}_{15}}\right]+C_{\mathcal{J}_{175}}^{I_{1} I_{2}} x_{12}^{\Delta_{175}^{(s)}} x_{12}^{\mu}\left[O_{\mu}^{\mathcal{J}_{175}}\right]+\ldots
\end{aligned}
$$

Here $R_{\mu}^{\mathcal{J}_{15}}$ is the R-symmetry current and $T_{\mu \nu}$ is the stress tensor of the full $\mathcal{N}=4$ $\mathrm{SYM}_{4}$ and $\Delta_{\mathcal{J}}^{(s)}$ is the anomalous dimension of the corresponding double-trace operator at strong coupling. The conformal blocks appearing in (3) encode all the strong coupling information for the anomalous dimensions and the couplings of the corresponding operators. In the place of an infinite number of single-trace operators in (2), (3) contains instead only three single-trace operators giving rise to the most singular terms. Note that the coefficients in front of the $R$-symmetry current and the stress tensor in (3) are different from the ones in (2). The reason is that the free-field operators $J_{\mu}^{\mathcal{J}_{15}}$ and $T_{\mu \nu}^{\text {free }}$ constructed only from scalars are split into operators belonging to different supersymmetry multiplets. Multiplets that are dual to string modes decouple in the strong coupling limit, while operators from the stress tensor multiplet are non-renormalized and show up at strong coupling.

The leading double-trace operators receive anomalous dimensions whose value at strong coupling was found to be

$$
\Delta_{O_{1}}^{(s)}=-\frac{16}{N^{2}}, \quad \Delta_{O_{15}}^{(s)}=-\frac{16}{N^{2}},
$$


while all the other operators shown in (3) have vanishing anomalous dimensions. The double trace operators in 84, 105 are in short multiplets and they are protected. The double-trace operator in $\mathbf{2 0}$ is not protected by unitarity and is allowed to acquire an anomalous dimension. Nevertheless, it was found to have vanishing anomalous dimension at strong coupling.

Comparison of the free-field and strong coupling OPEs (2) and (3) enabled us to make the predictions for the OPE structure at finite $N$ and $\lambda$ discussed in the Introduction. In the next Section we verify that these predictions are in agreement with the 2-loop 4-point function of CPOs. To this end we study the asymptotic behavior of the 4-point function in the direct channel $x_{12}^{2}, x_{34}^{2} \rightarrow 0$, which in terms of the "biharmonic ratios" $u=\frac{x_{12}^{2} x_{34}^{2}}{x_{13}^{2} x_{24}^{2}}, v=\frac{x_{12}^{2} x_{34}^{2}}{x_{14}^{2} x_{23}^{2}}$ and the variable $Y=1-\frac{v}{u}$, amounts to taking the short-distance limit $u, v, Y \rightarrow 0$. Our analysis closely follows [15] and is based on the knowledge of the conformal partial wave amplitudes of quasi-primary operators. In particular, consider the contributions to the OPE of two CPOs coming from a scalar, vector and second rank symmetric traceless tensor. Schematically this is given by

$$
\begin{aligned}
O^{I_{1}}\left(x_{1}\right) O^{I_{2}}\left(x_{2}\right)=C_{\mathcal{J}}^{I_{1} I_{2}} & \left(\frac{C_{O O S}}{C_{S}} \frac{1}{x_{12}^{4-\Delta_{S}}}\left[S^{\mathcal{J}}\right]-\frac{C_{O O T}}{C_{T}} \frac{x_{12}^{\mu} x_{12}^{\nu}}{x_{12}^{6-\Delta_{T}}}\left[T_{\mu \nu}^{\mathcal{J}}\right]\right. \\
& \left.+\frac{C_{O O V}}{C_{V}} \frac{x_{12}^{\mu}}{x_{12}^{5-\Delta_{V}}}\left[V_{\mu}^{\mathcal{J}}\right]+\ldots\right) .
\end{aligned}
$$

Here $\mathcal{J}$ denotes an index of an irrep of the $R$-symmetry group $S O(6), C_{\mathcal{J}}^{I_{1} I_{2}}$ are the Clebsch-Gordan coefficients and $\Delta_{S}, \Delta_{T}, \Delta_{V}$ are the conformal dimensions of the scalar, tensor and vector operators respectively. For any operator in the OPE, $C_{\mathcal{O}}$ and $C_{O O \mathcal{O}}$ denote the normalization constant in the 2-point function $\left\langle\mathcal{O}\left(x_{1}\right) \mathcal{O}\left(x_{2}\right)\right\rangle$ and the coupling constant in the three-point function $\left\langle O^{I}\left(x_{1}\right) O^{J}\left(x_{2}\right) \mathcal{O}\left(x_{3}\right)\right\rangle$, respectively. Then, the shortdistance expansion of the conformal partial amplitudes (CPWA) of the scalar $\mathrm{S}$, tensor $\mathrm{T}$ and vector $\mathrm{V}$ operators can be written as (c.f. [15])

$$
\begin{gathered}
\left\langle O^{I_{1}}\left(x_{1}\right) O^{I_{2}}\left(x_{2}\right) O^{I_{3}}\left(x_{3}\right) O^{I_{4}}\left(x_{4}\right)\right\rangle=\frac{C_{\mathcal{J}}^{I_{1} I_{2}} C_{\mathcal{J}}^{I_{3} I_{4}}}{x_{12}^{4} x_{34}^{4}} \\
\times\left[\frac{C_{O O S}^{2}}{C_{S}} v^{\frac{\Delta_{S}}{2}}\left(1+\frac{\Delta_{S}}{4} Y+\frac{\Delta_{S}^{3}}{16\left(\Delta_{S}-1\right)\left(\Delta_{S}+1\right)} v\left(1+\frac{\Delta_{S}+2}{4} Y\right)+\cdots\right)\right. \\
+\frac{C_{O O T}^{2}}{C_{T}} v^{\frac{\Delta_{T}}{2}}-1\left(\frac{1}{4} Y^{2}-\frac{1}{4} v-\frac{\Delta_{T}}{16} v Y \cdots\right) \\
\left.+\frac{C_{O O V}^{2}}{C_{V}} v^{\frac{\Delta_{V}-1}{2}}\left(\frac{1}{2} Y+\cdots\right)\right]
\end{gathered}
$$

where we assumed that $\Delta_{T}=4+\Delta_{T}^{(1)}$ and kept only terms linear in $\Delta_{T}^{(1)}$. The formulas for the leading contributions of a rank-2 traceless symmetric tensor and a vector can be 
generalized to the case of a rank- $l$ traceless symmetric tensor of dimension $\Delta_{l}$ and one gets a leading term of the form $v^{\frac{\Delta_{l}-l}{2}} Y^{l}$.

If we decompose the conformal dimension of an operator into a "canonical" part (equal to its free-field conformal dimension) and an "anomalous" part, taken to be a small parameter (see [15]), then (6) shows that the "anomalous dimensions" are related to terms of the form $v^{\frac{\Delta_{S}^{(0)}}{2}} \log v$ for scalar operators, $v^{\frac{\Delta_{V}^{(0)}-1}{2}} Y \log v$ for vector operators and $v^{\frac{\Delta_{T}^{(0)}-2}{2}} Y^{2} \log v$ for rank-2 tensor operators. Formula (6) is the basic tool in our analysis of the 2-loop 4-point function in Section 3 and the instanton contribution in Section 4.

\section{OPE analysis of the 2-loop 4-point function}

The 2-loop 4-point functions of the CPOs $\mathrm{O}_{2}$ were computed in [32-34] and the results obtained there can be represented in terms of a basic function $\Phi^{(1)}(v, u)$ that can be written in the form of a Mellin-Barnes integral as

$$
\Phi^{(1)}(x, y)=\frac{1}{(2 \pi \mathrm{i})^{2}} \int_{\mathcal{C}} \mathrm{d} s \mathrm{~d} t \Gamma^{2}(-s) \Gamma^{2}(-t) \Gamma^{2}(1+s+t) x^{s} y^{t},
$$

where the contour(s) $\mathcal{C}$ run parallel to the imaginary axis. Performing the integrations, we may cast it in a form suitable for studying the OPE as

$$
\begin{aligned}
\Phi^{(1)}(v, Y)=\sum_{n, m=0}^{\infty} & \frac{v^{n} Y^{m}}{(n !)^{2} m !} \frac{\Gamma^{2}(1+n) \Gamma^{2}(1+n+m)}{\Gamma(2+2 n+m)} \\
& \times[-\log v+2 \psi(2+2 n+m)-2 \psi(1+n+m)] .
\end{aligned}
$$

Then, the 4-point function of the CPOs $O^{I}$ reads

$$
\begin{aligned}
\left\langle O^{I_{1}}\left(x_{1}\right) O^{I_{2}}\left(x_{2}\right) O^{I_{3}}\left(x_{3}\right) O^{I_{4}}\left(x_{4}\right)\right\rangle & =\delta^{I_{1} I_{2}} \delta^{I_{3} I_{4}} a_{1}+\delta^{I_{1} I_{3}} \delta^{I_{2} I_{4}} a_{2}+\delta^{I_{1} I_{4}} \delta^{I_{2} I_{3}} a_{3} \\
+ & C^{I_{1} I_{2} I_{3} I_{4}} b_{2}+C^{I_{1} I_{3} I_{2} I_{4}} b_{1}+C^{I_{1} I_{3} I_{4} I_{2}} b_{3}
\end{aligned}
$$

where up to 2-loops the various coefficients are given by

$$
\begin{array}{ll}
a_{1}=\frac{1}{x_{12}^{4} x_{34}^{4}}\left[1-\frac{2 \tilde{\lambda}}{N^{2}} v \Phi^{(1)}(v, Y)\right], & b_{1}=\frac{4}{N^{2}} \frac{1}{x_{12}^{4} x_{34}^{4}}\left[v u-\frac{\tilde{\lambda}}{2} v(v u-v-u) \Phi^{(1)}(v, Y)\right], \\
a_{2}=\frac{1}{x_{12}^{4} x_{34}^{4}}\left[u^{2}-\frac{2 \tilde{\lambda}}{N^{2}} v u \Phi^{(1)}(v, Y)\right], & b_{2}=\frac{4}{N^{2}} \frac{1}{x_{12}^{4} x_{34}^{4}}\left[v+\frac{\tilde{\lambda}}{2} v(v+Y) \Phi^{(1)}(v, Y)\right], \\
a_{3}=\frac{1}{x_{12}^{4} x_{34}^{4}}\left[v^{2}-\frac{2 \tilde{\lambda}}{N^{2}} v^{2} \Phi^{(1)}(v, Y)\right], & b_{3}=\frac{4}{N^{2}} \frac{1}{x_{12}^{4} x_{34}^{4}}\left[u+\frac{\tilde{\lambda}}{2} \frac{v-Y}{1-Y} v \Phi^{(1)}(v, Y)\right] .
\end{array}
$$

Using the above result we may now study the OPE at 2-loops. We start with the projection into the singlet which includes important fields such as the stress tensor, the Konishi scalar and the double-trace operator $O_{1}$ with canonical dimension 4 . 


\subsection{Projection in the singlet}

Using the properly normalized projector in the singlet [15], we obtain for the first few terms in the short-distance expansion

$$
\begin{gathered}
\left.\left\langle O^{I_{1}}\left(x_{1}\right) O^{I_{2}}\left(x_{2}\right) O^{I_{3}}\left(x_{3}\right) O^{I_{4}}\left(x_{4}\right)\right\rangle\right|_{1}=\frac{\delta^{I_{1} I_{2}} \delta^{I_{3} I_{4}}}{x_{12}^{4} x_{34}^{4}}\left[1+\frac{4}{3 N^{2}} v\left(1+\frac{3 \tilde{\lambda}}{2} \log v-3 \tilde{\lambda}\right)\right. \\
+\frac{2}{3 N^{2}} v Y\left(1+\frac{3 \tilde{\lambda}}{2} \log v-\frac{3 \tilde{\lambda}}{2}\right)+\frac{2}{3 N^{2}} v Y^{2}\left(1+\frac{3 \tilde{\lambda}}{2} \log v-\frac{5 \tilde{\lambda}}{3}\right) \\
\left.+\frac{1}{10} v^{2}\left(1+\frac{2}{3 N^{2}}-\frac{2 \tilde{\lambda}}{N^{2}} \log v+\frac{230 \tilde{\lambda}}{45 N^{2}}\right)+\frac{1}{10} v^{2} Y\left(1+\frac{2}{3 N^{2}}-\frac{2 \tilde{\lambda}}{N^{2}} \log v+\frac{37 \tilde{\lambda}}{9 N^{2}}\right)\right] .
\end{gathered}
$$

The expansion (10) should be matched with the contributions coming from the first few low-dimensional operators in the singlet projection of the OPE (2). In the free-field theory limit the first fields which appear in the above OPE are the Konishi scalar $\mathcal{K}$ with free-field dimension 2, the stress tensor of 6 free scalar fields $T_{\mu \nu}^{\text {free }}$ and a double-trace operator $O_{1}^{\text {free }}$ with free-field dimension 4 . It is natural to assume that these are exactly the first few operators which appear also in the 2-loop OPE, allowing only for possible small corrections in their free-field dimensions and normalization constants in order to account for the logarithmic terms in (10). Although such an assumption seems to work for the Konishi scalar, it does not quite fit the 2-loop result (10) as there is a discrepancy in the coefficients in front of the stress tensor in free field theory and at 2-loops.

In order to properly interpret (10) one should realize that the stress tensor $T_{\mu \nu}$ expected to appear in it is different from $T_{\mu \nu}^{f r e e}$, since it receives contributions not only from the six scalars but also from the four Weyl fermions and the vector field of $\mathcal{N}=4 \mathrm{SYM}$. It has been argued in [44], following [45], that the general stress tensor of an interacting CFT involving scalars, fermions and vectors involves three, linearly independent and mutually orthogonal structures. ${ }^{5}$ For the specific case of $\mathcal{N}=4$ SYM, simple manipulations allow one to write the free-field stress tensor (i.e. the stress tensors of six massless free scalars) $T_{\mu \nu}^{f r e e}(x)$ as follows

$$
T_{\mu \nu}^{\text {free }}(x)=\frac{1}{5} T_{\mu \nu}(x)+\frac{10}{35} \mathcal{K}_{\mu \nu}(x)+\frac{18}{35} \Xi_{\mu \nu}(x),
$$

where the three structures depicted in (11) are mutually orthogonal and linearly independent. The idea of [44] is that the orthogonality and linear independence property is preserved by perturbation theory, i.e. the structures in (11) do not mix under renormalization. The (symmetric and traceless) tensor $\mathcal{K}_{\mu \nu}$ belongs to the Konishi supermultiplet

\footnotetext{
${ }^{5}$ This is easily seen in free-field theory, where the stress tensors for free scalars, fermions and vectors provide three linearly independent and orthogonal to each other structures [45].
} 
while the (symmetric and traceless) $\Xi_{\mu \nu}$ is the lowest component of a new supermultiplet. The full stress tensor $T_{\mu \nu}$ is expected to remain conserved, therefore, it retains its canonical dimension at any order in perturbation theory. However, $\mathcal{K}_{\mu \nu}$ and $\Xi_{\mu \nu}$ can, and do, acquire anomalous dimensions.

We preface the more detailed analysis of the OPE with some necessary comments. The fact that $T_{\mu \nu}$ is canonically normalized allows one to find the free-field value of the normalization constants of the 2-point functions of the three operators in (11) as

$$
C_{T}=5 C_{t}, \quad C_{\mathcal{K}_{1}}=\frac{7}{2} C_{t}, \quad C_{\Xi}=\frac{35}{18} C_{t},
$$

where $C_{t}=32 \tilde{\lambda}^{2}$ is a normalization constant for $T_{\mu \nu}^{\text {free }}$. The value of the coupling $C_{O O T}=$ $\frac{16 \tilde{\lambda}}{3 N}$ is fixed by the conformal Ward identity. The free-field theory OPE (2) together with (11) and (12) gives the free-field value of the normalization constants

$$
C_{O O \mathcal{K}_{1}}=C_{O O \Xi}=\frac{16 \tilde{\lambda}}{3 N}
$$

Recall that the Konishi field is canonically normalized, i.e. $C_{\mathcal{K}}=1$ and the free-field result for $C_{O O \mathcal{K}}$ is $C_{O O \mathcal{K}}=\frac{2}{3^{1 / 2} N^{2}}$.

In the sequel we assume that for any operator $\mathcal{O}$ in the OPE the ratio $\frac{C_{O O O}}{C_{\mathcal{O}}}$ is kept equal to its free-field value. The correction to a coupling dependent normalization constant $C_{O O O}(\tilde{\lambda})$ is introduced in the following way

$$
C_{O O \mathcal{O}}(\tilde{\lambda})=C_{O O \mathcal{O}}\left(1+C_{O O \mathcal{O}}^{(1)}\right)
$$

where $C_{O O O}$ stands for the free-field value.

Now taking into account the splitting (11), using (6) for the contributions of scalars and symmetric traceless tensors to the OPE and expanding the anomalous dimensions and the normalization constants, we find for the leading terms of the short-distance expansion of the singlet projection

$$
P_{\text {singlet }}^{O P E}=\frac{\delta^{I_{1} I_{2}} \delta^{I_{3} I_{4}}}{x_{12}^{4} x_{34}^{4}}\left[1+A_{10} v+A_{11} v Y+A_{12} v Y^{2}\right],
$$

where the coefficients $A$ are given by

$$
\begin{aligned}
A_{10} & =\frac{C_{O O \mathcal{K}}^{2}}{C_{\mathcal{K}}}\left[1+\frac{\eta_{\mathcal{K}}}{2} \log v+C_{O O \mathcal{K}}^{(1)}\right] \\
A_{11} & =\frac{1}{2} \frac{C_{O O \mathcal{K}}^{2}}{C_{\mathcal{K}}}\left[1+\frac{\eta_{\mathcal{K}}}{2} \log v+\frac{\eta_{\mathcal{K}}}{2}+C_{O O \mathcal{K}}^{(1)}\right] \\
A_{12} & =\frac{1}{3} \frac{C_{O O \mathcal{K}}^{2}}{C_{\mathcal{K}}}\left[1+\frac{\eta_{\mathcal{K}}}{2} \log v+C_{O O \mathcal{K}}^{(1)}+\frac{2}{3} \eta_{\mathcal{K}}\right]+\frac{1}{4} \frac{C_{O O T}^{2}}{C_{T}} \\
& +\frac{1}{4} \frac{C_{O O \mathcal{K}_{\mathbf{1}}}^{2}}{C_{\mathcal{K}_{1}}}\left[1+\frac{1}{2} \eta_{\mathcal{K}_{\mathbf{1}}} \log v+C_{O O \mathcal{K}_{\mathbf{1}}}^{(1)}\right]+\frac{1}{4} \frac{C_{O O \Xi}^{2}}{C_{\Xi}}\left[1+\frac{1}{2} \eta_{\Xi} \log v+C_{O O \Xi}^{(1)}\right] .
\end{aligned}
$$


Here the parameters $\eta_{\mathcal{K}}, \eta_{\mathcal{K} \mathbf{1}}$ and $\eta_{\Xi}$ correspond to the small corrections to the canonical dimensions of the operators $\mathcal{K}, \mathcal{K}_{\mu \nu}$ and $\Xi_{\mu \nu}$, respectively, while $C_{O O \mathcal{K}}^{(1)}, C_{O O \mathcal{K}_{1}}^{(1)}$ and $C_{O O \Xi}^{(1)}$ denote the small corrections to the corresponding free-field normalization constants. According to the discussion above the free-field values of the ratios of the 3- and 2-point normalization constants occurring in (15)-(17) are given by

$$
\frac{C_{O O \mathcal{K}}^{2}}{C_{\mathcal{K}}}=\frac{4}{3 N^{2}}, \quad \frac{C_{O O \mathcal{K}_{1}}^{2}}{C_{\mathcal{K}_{\mathbf{1}}}}=\frac{16}{63 N^{2}}, \quad \frac{C_{O O \Xi}^{2}}{C_{\Xi}}=\frac{16}{35 N^{2}} .
$$

Requiring consistency of the terms carrying equal powers of $v$ and $Y$ in (10) and (14) we then obtain the anomalous dimensions and corrections to the coupling constants of the operators discussed above.

Consistency of the terms proportional to $v$ in (10) and (14) gives

$$
\eta_{\mathcal{K}}=3 \tilde{\lambda}, \quad C_{O O \mathcal{K}}^{(1)}=-3 \tilde{\lambda}
$$

The value of $\eta_{\mathcal{K}}$ coincides with the well-known value for the 2-loop anomalous dimension for the Konishi scalar [44].

Using the result (19), we immediately see that the terms proportional to $v Y$ in $(10)$ and (14) are consistent.

Consistency of the terms proportional to $v Y^{2}$ in (10) and (14) gives

$$
\begin{aligned}
\frac{3}{2} \tilde{\lambda} & =\frac{1}{7} \eta_{\mathcal{K}_{1}}+\frac{9}{35} \eta_{\Xi}, \\
-\frac{3}{2} \tilde{\lambda} & =\frac{1}{7} C_{O O \mathcal{K}_{1}}^{(1)}+\frac{9}{35} C_{O O \Xi}^{(1)} .
\end{aligned}
$$

This shows that the consistency of the short-distance expansion with the OPE is not sufficient to determine the individual anomalous dimensions and corrections to the couplings of the split fields $\mathcal{K}_{\mu \nu}$ and $\Xi_{\mu \nu}$. However, here comes the input of supersymmetry which rectifies the situation. Namely, requiring that $\mathcal{K}_{\mu \nu}$ is in the same supermultiplet as the Konishi scalar $\mathcal{K}$ we fix its anomalous dimension to be the same as $\mathcal{K}$

$$
\eta_{\mathcal{K}_{1}}=3 \tilde{\lambda}
$$

Then, we easily find from (20) that

$$
\eta_{\Xi}=\frac{25}{6} \tilde{\lambda}
$$

in complete agreement with [44]. 
The terms in (10) proportional to $v^{2}$ and $v^{2} Y$ encode the information about scalar operators of free-field dimension 4. Recall that in free-field theory the corresponding terms match with the contribution of a unique operator

$$
O_{1}^{\text {free }}=\frac{1}{40 \tilde{\lambda}^{2}}\left(: \operatorname{tr}\left(\phi^{i} \phi^{j}\right) \operatorname{tr}\left(\phi^{i} \phi^{j}\right):-\frac{1}{6}: \operatorname{tr}\left(\phi^{i} \phi^{i}\right) \operatorname{tr}\left(\phi^{j} \phi^{j}\right):\right)
$$

with the 2-point function

$$
\left\langle O_{1}^{\text {free }}\left(x_{1}\right) O_{1}^{\text {free }}\left(x_{2}\right)\right\rangle=\frac{1}{10}\left(1+\frac{2}{3 N^{2}}\right) \frac{1}{x_{12}^{8}},
$$

while at strong coupling the singlet channel was shown [15] to contain a scalar operator $O_{1}$ of approximate dimension 4 with the following 2-point function:

$$
\left\langle O_{1}\left(x_{1}\right) O_{1}\left(x_{2}\right)\right\rangle=\frac{1}{10}\left(1+\frac{38}{15 N^{2}}\right) \frac{1}{x_{12}^{8+2 \Delta_{1}^{(s)}}} .
$$

Although the difference of 2-point functions of $O_{1}^{\text {free }}$ and $O_{1}$ might be explained by the fact that they are computed in different regimes and the operator is not protected, it is more natural to assume that $O_{1}^{\text {free }}$ splits in perturbation theory into a sum of operators such that only one of them is dual to a gravity state. Indeed, in free-field theory one finds a number of linearly independent operators of dimension 4, e.g., : $\operatorname{tr}\left(\phi^{i} \phi^{j}\right) \operatorname{tr}\left(\phi^{i} \phi^{j}\right)$ :, $: \operatorname{tr}\left(\phi^{i} \phi^{i}\right) \operatorname{tr}\left(\phi^{j} \phi^{j}\right):$ and $: \operatorname{tr}\left(\phi^{i} \phi^{j} \phi^{i} \phi^{j}\right)$ : that may mix under renormalization. To find the individual anomalous dimensions at two loops one should diagonalize their mixing matrix. We then expect to find a unique operator $O_{1}$ (dual to a "two-particle" gravity state), whose anomalous dimension behaves as $\frac{\tilde{\lambda}}{N^{2}}$, while the other operators (dual to string modes) should have the anomalous dimensions of the Konishi type $\sim \tilde{\lambda}$. Such a splitting, similar in spirit with the above discussed splitting of the stress tensor, seems to be necessary in order to account for the fact that at strong coupling we find only one operator with approximate dimension 4 while at any order in perturbation theory we expect an operator mixing. However, the knowledge of the correlation functions of CPOs alone is not sufficient in order to establish the mixing matrix and additional information is needed, e.g. the knowledge of correlation functions of four Konishi scalars or other operators.

\subsection{Projection in $\mathbf{2 0}$}

Projecting the 4-point function in the $\mathbf{2 0}$ irrep we obtain for the leading in $v, Y$ terms the following answer

$$
\left.\left\langle O^{I_{1}}\left(x_{1}\right) O^{I_{2}}\left(x_{2}\right) O^{I_{3}}\left(x_{3}\right) O^{I_{4}}\left(x_{4}\right)\right\rangle\right|_{\mathbf{2 0}}=\frac{C_{\mathcal{J}_{20}}^{I_{1} I_{2}} C_{\mathcal{J}_{20}}^{I_{3} I_{4}}}{x_{12}^{4} x_{34}^{4}}\left[\frac{40}{3 N^{2}} v\left(1+\frac{1}{2} Y+\frac{1}{2} Y^{2}\right)+\right.
$$




$$
\left.+v^{2}\left(2+\frac{2}{3 N^{2}}\right)(1+Y)-\frac{\tilde{\lambda}}{N^{2}} \frac{20}{3} v\left(Y^{2}-v-\frac{3}{4} v Y\right)+\frac{\tilde{\lambda}}{N^{2}} \frac{10}{3} v\left(Y^{2}-v-v Y\right) \log v\right] .
$$

According to our discussion of the free-field theory OPE in Section 2, the first three low-dimension operators contributing to 20 are the CPOs themselves, the double-trace operator $O_{\mathbf{2 0}}$, and a symmetric second rank tensor $\mathcal{K}_{\mu \nu}^{I} \equiv \mathcal{K}_{\mathbf{2 0}}$ of approximate dimension 4. On the other hand at strong coupling and in the large $N$ limit we found that only the CPOs and the $\mathrm{O}_{\mathbf{2 0}}$ survive and keep their free-field dimension. While the non-renormalization property of CPO is well-known, the non-renormalization of $\mathrm{O}_{\mathbf{2 0}}$ is a new phenomenon that cannot be explained on the basis of unitarity. A natural suggestion made in [15] is that $O_{\mathbf{2 0}}$ is non-renormalized in perturbation theory at finite $N$. As far as $\mathcal{K}_{\mathbf{2 0}}$ is concerned, being dual to a string mode it receives perturbatively large anomalous dimension and decouples from the spectrum at strong coupling. ${ }^{6}$ Let us see how this picture is compatible with two-loop result (26).

The last two terms in (26) are proportional to $\tilde{\lambda}$ and we interpret them as loop contribution to the coupling $C_{O O \mathcal{K}_{\mathbf{2}}}$ and to the anomalous dimension of $\mathcal{K}_{\mathbf{2 0}}$ respectively. Indeed, if we denote the anomalous dimension of $\mathcal{K}_{\mathbf{2 0}}$ as $\Delta_{\mathcal{K}_{\mathbf{2 0}}}^{(1)}$ then the $\log v$-dependent term in (26) occurs due to the contribution of the conformal block of the second rank tensor with free-field dimension 4 (c.f. (6)). Therefore, the other two operators, CPO and $\mathrm{O}_{\mathbf{2 0}}$, do not receive anomalous dimensions. To compute $\Delta_{\mathcal{K}_{\mathbf{2 0}}}^{(1)}$ one needs to know the freefield value of the ratio $\frac{C_{O O \mathcal{K}_{20}}^{2}}{C_{\mathcal{K}_{20}}}$. This can be found by considering, e.g., the $\tilde{\lambda}$-independent $v Y^{2}$ terms in (26). By using the CPWA of the scalar with dimension 2 and comparing $v Y^{2}$ terms in (6) with the ones in (26) one gets

$$
\frac{C_{O O O}^{2}}{3 C_{O}}+\frac{C_{O O \mathcal{K}_{\mathbf{2 0}}}^{2}}{4 C_{\mathcal{K}_{\mathbf{2 0}}}}=\frac{20}{3 N^{2}}
$$

Since $\frac{C_{O O O}^{2}}{C_{O}}=\frac{40}{3 N^{2}}[15]$ one finds the following free-field value

$$
\frac{C_{O O \mathcal{K}_{20}}^{2}}{C_{\mathcal{K}_{20}}}=\frac{80}{9 N^{2}}
$$

Analogously, analysis of the $v^{2}$ terms in (6) and in (26) produces the free-field relation

$$
\frac{C_{O O O}^{2}}{6 C_{O}}+\frac{C_{O O O_{\mathbf{2 0}}}^{2}}{C_{O_{\mathbf{2 0}}}}-\frac{C_{O O \mathcal{K}_{\mathbf{2 0}}}^{2}}{4 C_{\mathcal{K}_{\mathbf{2 0}}}}=2+\frac{2}{3 N^{2}}
$$

that further gives

$$
\frac{C_{\mathrm{OOO}_{20}}^{2}}{C_{\mathrm{O}_{20}}}=2+\frac{2}{3 N^{2}}
$$

\footnotetext{
${ }^{6}$ In principle one could expect a splitting of $\mathcal{K}_{\mathbf{2 0}}$ into a sum of operators dual to string modes. However, our analysis will show that this does not happen.
} 
Note that the same answer was found by studying the 4-point function at strong coupling [15], that agrees with the conjectured non-renormalization of the operator $O_{\mathbf{2 0}}$.

Now the $\log v$-dependent term allows to find $\Delta_{\mathcal{K}_{\mathbf{2 0}}}^{(1)}: \frac{C_{O O \mathcal{K}_{\mathbf{2 0}}}^{2}}{8 C_{\mathcal{K}_{\mathbf{2 0}}}} \Delta_{\mathcal{K}_{\mathbf{2 0}}}^{(1)}=\frac{10 \tilde{\lambda}}{3 N^{2}}$, i.e. ,

$$
\Delta_{\mathcal{K}_{\mathbf{2 0}}}^{(1)}=3 \tilde{\lambda}
$$

Thus, the anomalous dimension of $\mathcal{K}_{\mathbf{2 0}}$ is the same as the dimension of the Konishi field, hence they are from the same multiplet.

Finally the $\tilde{\lambda}$-dependent terms without $\log v$ are due to the loop correction to the freefield value of $C_{O O \mathcal{K}_{\mathbf{2 0}}}$. Indeed, the $\tilde{\lambda} v Y^{2}$ term in (26) allows one to find $C_{O O \mathcal{K}_{\mathbf{2 0}}}^{(1)}=-3 \tilde{\lambda}$. We can check the consistency of the assumption that there is only one tensor operator in the $\mathbf{2 0}$ which receives corrections to its anomalous dimension and structure constant. To this end we compute the term of order $v^{2} Y$ by using the found anomalous dimension and correction to the structure constant, and see that it coincides with the corresponding term in (26).

\subsection{Projection in $\mathbf{8 4}$}

Projecting in $\mathbf{8 4}$ we get for the leading terms in the short-distance expansion

$$
\begin{gathered}
\left.\left\langle O^{I_{1}}\left(x_{1}\right) O^{I_{2}}\left(x_{2}\right) O^{I_{3}}\left(x_{3}\right) O^{I_{4}}\left(x_{4}\right)\right\rangle\right|_{\mathbf{8 4}}=\frac{C_{\mathcal{J}_{84}}^{I_{1} I_{2}} C_{\mathcal{J}_{84}}^{I_{3} I_{4}}}{x_{12}^{4} x_{34}^{4}}\left[\frac{6 \tilde{\lambda}}{N^{2}} v^{2}(1+Y) \log v\right. \\
\left.+v^{2}\left(\left(2-\frac{2}{N^{2}}\right)(1+Y)-\frac{12 \tilde{\lambda}}{N^{2}}-\frac{9 \tilde{\lambda}}{N^{2}} Y\right)\right] .
\end{gathered}
$$

A strong coupling result suggests that at finite $\lambda$ the OPE of CPOs contains two operators $O_{\mathbf{8 4}}$ and $\mathcal{K}_{\mathbf{8 4}}$ transforming in the irrep $\mathbf{8 4}$. The operator $O_{\mathbf{8 4}}$ has protected both the dimension and the normalization constants of the 2- and 3-point functions, while $\mathcal{K}_{\mathbf{8 4}}$ is from the Konishi multiplet and receives anomalous dimension.

At zeroth order in $\tilde{\lambda}$, the non-logarithmic term in (30) gives for the free-field values of the normalization constants ${ }^{7}$

$$
C_{\mathrm{O}_{\mathbf{8 4}}}+\frac{1}{N^{2}} C_{\mathcal{K}_{\mathbf{8 4}}}=2-\frac{2}{N^{2}}
$$

\footnotetext{
${ }^{7}$ We exhibit a coefficient $1 / N^{2}$ in front of $C_{\mathcal{K}_{\mathbf{8 4}}}$ to emphasize the fact that a free-field operator $O^{f r}$ undergoes a splitting into a sum of operators as $O^{f r}=O^{g r}+\frac{1}{N} O^{s t r}$, where $O^{g r}$ is dual to a supergravity two-particle state and $O^{\text {str }}$ dual to a string state [15]. The same splitting applies to operators in irrep 175.
} 
The constant $C_{O_{84}}$ is non-renormalized and is found [15] to be $C_{O_{84}}=2-\frac{6}{N^{2}}$. Therefore, $C_{\mathcal{K}_{\mathbf{8 4}}}=4$. The $\log v$ term in (30) allows one to read off the anomalous dimension $\Delta_{\mathcal{K}_{\mathbf{8 4}}}^{(1)}$ of $\mathcal{K}_{\mathbf{8 4}}: \Delta_{\mathcal{K}_{\mathbf{8 4}}}^{(1)}=3 \tilde{\lambda}$, as it should be for the member of the Konishi multiplet. Finally, from the $\tilde{\lambda} v^{2}$ term in (30) we can find a correction $C_{O O \mathcal{K}_{\mathbf{8 4}}}^{(1)}=-3 \tilde{\lambda}$.

\subsection{Projection in $\mathbf{1 0 5}$}

For the leading terms of the projection of the 4-point function in $\mathbf{1 0 5}$ we find

$$
\begin{aligned}
& \left.\left\langle O^{I_{1}}\left(x_{1}\right) O^{I_{2}}\left(x_{2}\right) O^{I_{3}}\left(x_{3}\right) O^{I_{4}}\left(x_{4}\right)\right\rangle\right|_{\mathbf{1 0 5}}=\frac{C_{\mathcal{J}_{105}}^{I_{1} I_{2}} C_{\mathcal{J}_{105}}^{I_{3} I_{4}}}{x_{12}^{4} x_{34}^{4}}\left[\frac{2 \tilde{\lambda}}{N^{2}} v^{3}\left(1+\frac{3}{2} Y\right) \log v\right. \\
& \left.\quad+v^{2}\left(2+\frac{4}{N^{2}}\right)(1+Y)\right] .
\end{aligned}
$$

The last formula shows that the first $\log v$-term appears at order $v^{3}$. Therefore, all symmetric traceless rank-2k tensor operators of dimension $4+2 k$ transforming in the $\mathbf{1 0 5}$ have protected conformal dimensions, the lowest operator among them is the double-trace operator $O_{\mathbf{1 0 5}}$. The $\log v$-term in (31) indicates the appearance of the anomalous dimensions for the symmetric traceless rank- $2 k$ tensors of the canonical dimensions $6+2 k$. However, at strong coupling the first $\log v$-term appears only at order $v^{4}$ (see (4.16) of [15]). Thus, a free-field tensor operator of dimension $6+2 k$ undergoes a splitting into two operators, one has a protected dimension and normalization constants, another one receives perturbatively an anomalous dimension and disappears at strong coupling.

\subsection{Projection in $\mathbf{1 5}$}

Here we comment briefly on the projection in the irrep 15 whose leading terms have the form

$$
\left.\left\langle O^{I_{1}}\left(x_{1}\right) O^{I_{2}}\left(x_{2}\right) O^{I_{3}}\left(x_{3}\right) O^{I_{4}}\left(x_{4}\right)\right\rangle\right|_{15}=\frac{C_{\mathcal{J}_{15}}^{I_{1} I_{2}} C_{\mathcal{J}_{15}}^{I_{3} I_{4}}}{x_{12}^{4} x_{34}^{4}}\left[\frac{8}{N^{2}} v Y-\frac{16 \tilde{\lambda}}{N^{2}} v Y+\frac{8 \tilde{\lambda}}{N^{2}} v Y \log v\right] .
$$

The presence on the term $v Y \log v$ shows the appearance of the anomalous dimension for the vector operator $\mathcal{K}_{\mu}^{\mathcal{J}_{15}}$ of dimension 3. At strong coupling, however, the term $v Y \log v$ is absent and the dimension 3 operator which is the $R$-symmetry current $R_{\mu}^{\mathcal{J}_{15}}$ has protected conformal dimension. Thus, at finite $\lambda$ the contribution to $\mathbf{1 5}$ comes from two operators, $\mathcal{K}_{\mu}^{\mathcal{J}_{15}}$ and $R_{\mu}^{\mathcal{J}_{15}}$. From the $\tilde{\lambda}$-independent term $v Y$ in (32) we read off the relation for the free-field values of ratios of the normalization constants of these operators:

$$
\frac{C_{O O \mathcal{K}_{\mu}}^{2}}{2 C_{\mathcal{K}_{\mu}}}+\frac{C_{O O R_{\mu}}^{2}}{2 C_{R_{\mu}}}=\frac{8}{N^{2}} .
$$


Taking into account that $\frac{C_{O O R_{\mu}}^{2}}{2 C_{R_{\mu}}}=\frac{8}{3 N^{2}}$ we find

$$
\frac{C_{O O \mathcal{K}_{\mu}}^{2}}{2 C_{\mathcal{K}_{\mu}}}=\frac{16}{3 N^{2}}
$$

Finally, from the $v Y \log v$ term one obtains the anomalous dimension for $\mathcal{K}_{\mu}^{\mathcal{J}_{15}}$ :

$$
\Delta_{\mathcal{K}_{\mu}}^{(1)}=3 \tilde{\lambda}
$$

i.e. the vector operator $\mathcal{K}_{\mu}^{\mathcal{J}_{15}}$ is in the Konishi multiplet.

\subsection{Projection in $\mathbf{1 7 5}$}

Finally, projecting the 4-point function on irrep 175 one obtains for the leading terms the following expression:

$$
\left.\left\langle O^{I_{1}}\left(x_{1}\right) O^{I_{2}}\left(x_{2}\right) O^{I_{3}}\left(x_{3}\right) O^{I_{4}}\left(x_{4}\right)\right\rangle\right|_{\mathbf{1 7 5}}=\frac{C_{\mathcal{J}_{175}}^{I_{1} I_{2}} C_{\mathcal{J}_{175}}^{I_{3} I_{4}}}{x_{12}^{4} x_{34}^{4}}\left[2 v^{2} Y-\frac{4 \tilde{\lambda}}{N^{2}} v^{2} Y+\frac{2 \tilde{\lambda}}{N^{2}} v^{2} Y \log v\right]
$$

At strong coupling the first $\log v$ term occurs at order $v^{3} Y$ (c.f. Section 4.6 of [15]), while here it appears at order $v^{2} Y$. Thus, at finite $\lambda$ the contribution of the lowest dimension operators to the irrep. 175 comes from two operators $\mathcal{K}_{\mathbf{1 7 5}}$ and $O_{\mathbf{1 7 5}}$, both with approximate dimension 5 . The first operator receives infinite anomalous dimension at strong coupling, while the second one is non-renormalized due to the shortening condition [31]. The $v^{2} Y$ term in (36) produces for the free-field constants the following relation

$$
\frac{1}{N^{2}} C_{O O \mathcal{K}_{175}}+C_{O O O}{ }_{175}=4
$$

As was found in [15] $C_{O O O_{175}}=4-\frac{8}{3 N^{2}}$ and, therefore,

$$
C_{O O \mathcal{K}_{175}}=\frac{8}{3}
$$

Then the $\log v$ term allows one to find $\Delta_{\mathcal{K}_{\mathbf{1 7 5}}}^{(1)}=3 \tilde{\lambda}$ justifying thereby that $\mathcal{K}_{\mathbf{1 7 5}}$ belongs to the Konishi multiplet.

\section{Instanton contribution}

To analyze the instanton contribution to the 4-point function of the lowest weight CPOs we use the results of [38-41]. Firstly, we follow [42] to write the 4-point function 
of the CPOs (2) as

$$
\begin{aligned}
\left\langle\phi^{i_{1} j_{1}}\left(x_{1}\right) \phi^{i_{2} j_{2}}\left(x_{2}\right) \phi^{i_{3} j_{3}}\left(x_{3}\right) \phi^{i_{4} j_{4}}\left(x_{4}\right)\right\rangle & =a_{1}(s, t) \frac{\delta_{\left\{i_{1} j_{1}\right\}}^{i_{2} j_{2}} \delta_{\left\{i_{3} j_{3}\right\}}^{i_{4} j_{4}}}{x_{12}^{4} x_{34}^{4}} \\
& +a_{2}(s, t) \frac{\delta_{\left\{i_{1} j_{1}\right\}}^{i_{3} j_{3}} \delta_{\left\{i_{2} j_{2}\right\}}^{i_{4} j_{4}}}{x_{13}^{4} x_{24}^{4}}+a_{3}(s, t) \frac{\delta_{\left\{i_{1} j_{1}\right\}}^{i_{4} j_{4}} \delta_{\left\{i_{2} j_{2}\right\}}^{i_{3} j_{3}}}{x_{14}^{4} x_{23}^{4}} \\
& +b_{1}(s, t) \frac{\delta_{\left\{i_{1} j_{1}\right\}\left\{i_{2} j_{2}\right\}}^{\left\{i_{3}\left\{j_{4} i_{4}\right\} j_{3}\right\}}}{x_{13}^{2} x_{14}^{2} x_{23}^{2} x_{24}^{2}}+b_{2}(s, t) \frac{\delta_{\left\{i_{1} j_{1}\right\}\left\{i_{3} j_{3}\right\}}^{\left.i_{2}\left\{j_{4} i_{4}\right\} j_{2}\right\}}}{x_{12}^{2} x_{14}^{2} x_{23}^{2} x_{34}^{2}} \\
& +b_{3}(s, t) \frac{\delta_{\left\{i_{1} j_{1}\right\}\left\{i_{4} j_{4}\right\}}^{\left.2 i_{2}\left\{j_{3} i_{3}\right\} j_{2}\right\}}}{x_{12}^{2} x_{13}^{2} x_{24}^{2} x_{34}^{2}}
\end{aligned}
$$

where $\phi^{i j}=\frac{1}{2^{1 / 2} \hat{\lambda}} \operatorname{tr}\left[\phi^{i} \phi^{j}-\frac{1}{6} \delta^{i j} \phi^{2}\right], i, j=1,2, . ., 6$ and

$$
s=\frac{x_{12}^{2} x_{34}^{2}}{x_{13}^{2} x_{24}^{2}}, \quad t=\frac{x_{14}^{2} x_{23}^{2}}{x_{13}^{2} x_{24}^{2}} .
$$

The traces are over $S U(N)$ adjoint indices and the $S O(6)$ group-theoretic $\delta$-factors in (39) are products of Kroenecker $\delta^{\prime} s$ (c.f. [42]).

Superconformal invariance implies that (39) is actually determined in terms of only two arbitrary functions (e.g. $a_{1}$ and $b_{2}$ ) of $s$ and $t$. This fact allow us to restore the instanton contribution to the full 4-point function (39) from the results of [38-41] as follows. In the $\mathcal{N}=2$ formulation of $\mathcal{N}=4$ the six scalars in the fundamental of $S O(6)$ are decomposed in one complex scalar $\varphi$ and four scalars comprising the $\mathcal{N}=2$ matter hypermultiplet. The complex scalar is $\varphi=\phi^{5}+i \phi^{6}$ and one defines

$$
\operatorname{tr}\left(\varphi^{2}\right)=\operatorname{tr}\left(\phi^{55}\right)-\operatorname{tr}\left(\phi^{66}\right)+2 i \operatorname{tr}\left(\phi^{56}\right)=Y^{i j} \operatorname{tr}\left(\phi^{i} \phi^{j}\right)
$$

where $Y^{i j}=\delta^{i 5} \delta^{j 5}-\delta^{i 6} \delta^{j 6}+\mathrm{i}\left(\delta^{i 5} \delta^{j 6}+\delta^{i 6} \delta^{j 5}\right)$. Then, from (39) using the nilpotency of $Y^{i j}$ we obtain

$$
\begin{aligned}
\left\langle\operatorname{tr}\left(\varphi^{2}\right)\left(x_{1}\right) \operatorname{tr}\left(\varphi^{2}\right)\left(x_{2}\right) \operatorname{tr}\left(\bar{\varphi}^{2}\right)\left(x_{3}\right) \operatorname{tr}\left(\bar{\varphi}^{2}\right)\left(x_{4}\right)\right\rangle & =a_{2}(s, t) \frac{16}{x_{13}^{4} x_{24}^{4}}+a_{3}(s, t) \frac{16}{x_{14}^{4} x_{23}^{4}} \\
& +b_{1}(s, t) \frac{16}{x_{13}^{2} x_{14}^{2} x_{23}^{2} x_{24}^{2}} .
\end{aligned}
$$

The last correlator is precisely the one computed in [38-41] and the result reads (omitting the anti-instanton contributions)

$$
\left\langle\operatorname{tr}\left(\varphi^{2}\right)\left(x_{1}\right) \operatorname{tr}\left(\varphi^{2}\right)\left(x_{2}\right) \operatorname{tr}\left(\bar{\varphi}^{2}\right)\left(x_{3}\right) \operatorname{tr}\left(\bar{\varphi}^{2}\right)\left(x_{4}\right)\right\rangle=16 Q x_{12}^{4} x_{34}^{4} D_{4444}\left(x_{1}, x_{2}, x_{3}, x_{4}\right),
$$

where the $D$-functions are defined in [15] and we have absorbed all the normalization factors into $Q$ defined as

$$
Q=\frac{1}{4 \tilde{\lambda}^{4}} \cdot \frac{\sqrt{N} g_{Y M}^{8}}{2^{33} \pi^{27 / 2}} k^{1 / 2} e^{2 \pi i k \tau} \sum_{d \mid k} \frac{1}{d^{2}} \cdot \frac{2^{30} \cdot 3^{4}}{16},
$$


with $\tau$ the usual complex Yang-Mills coupling. The first factor in (44) is due to the normalization of the CPOs, the second factor comes from the $k$-instanton measure in the large- $N$ limit and also takes into account the $R$-weight of the CPOs and the last one is the result of the integration of the fermionic zero modes. $Q$ is not a modular invariant function, it is only the leading term of the modular invariant expression in the large $g_{Y M}$-limit.

In order now to read off the functions $a_{1}, a_{3}$ and $b_{2}$ in (42) from (43) we can exploit the result of [42] according to which the above three functions are in fact expressed in terms of only one function $\mathcal{F}(s, t)$ as

$$
b_{1}(s, t)=(s-t-1) \mathcal{F}(s, t), a_{2}(s, t)=\mathcal{F}(s, t), a_{3}(s, t)=t \mathcal{F}(s, t) .
$$

The function $\mathcal{F}(s, t)$ should satisfy the following crossing-symmetry properties

$$
\mathcal{F}(s, t)=\mathcal{F}(t, s)=\frac{1}{t} \mathcal{F}(s / t, 1 / t) .
$$

From (42) and (45) we then obtain

$$
\mathcal{F}(s, t)=Q \frac{s}{t^{3}} D(s, t)=Q \frac{v^{3}}{u^{2}} \bar{D}_{4444}(v, Y)
$$

where

$$
\begin{aligned}
D(s, t) & =\bar{D}_{4444}(s, t) \\
& =2 K \int \mathrm{d} t_{1} \ldots \mathrm{d} t_{4}\left(t_{1} t_{2} t_{3} t_{4}\right)^{3} \exp \left[-t_{1}\left(t_{2}+t_{3}+t_{4}\right)-t_{2} t_{3}-\frac{1}{t} t_{2} t_{4}-\frac{s}{t} t_{3} t_{4}\right]
\end{aligned}
$$

and $K$ was defined in [15]. Using the above integral representation it is easy to check that the function $\mathcal{F}(s, t)$ does satisfy the relations $(46)$.

Multiplying (39) with $C_{i j}^{I}$ we find the instanton contribution to the complete 4-point function of the lowest weight CPOs as

$$
\begin{gathered}
\left.\left\langle O^{I_{1}}\left(x_{1}\right) O^{I_{2}}\left(x_{2}\right) O^{I_{3}}\left(x_{3}\right) O^{I_{4}}\left(x_{4}\right)\right\rangle\right|_{\text {inst }}=\frac{1}{x_{12}^{4} x_{34}^{4}}\left[\delta^{I_{1} I_{2}} \delta^{I_{3} I_{4}} A_{1}(v, Y)+\delta^{I_{1} I_{3}} \delta^{I_{2} I_{4}} A_{2}(v, Y)\right. \\
\left.+\delta^{I_{1} I_{4}} \delta^{I_{2} I_{3}} A_{3}(v, Y)+C^{I_{1} I_{2} I_{3} I_{4}} B_{2}(v, Y)+C^{I_{1} I_{3} I_{2} I_{4}} B_{1}(v, Y)+C^{I_{1} I_{3} I_{4} I_{2}} B_{3}(v, Y)\right], \quad(49)
\end{gathered}
$$

where

$$
\begin{aligned}
& A_{1}(v, Y)=a_{1}(s, t)=Q \frac{v^{3}}{u} \bar{D}_{4444}(v, Y) \\
& A_{2}(v, Y)=u^{2} a_{2}(s, t)=Q v^{3} \bar{D}_{4444}(v, Y)
\end{aligned}
$$




$$
\begin{aligned}
& A_{3}(v, Y)=v^{2} a_{3}(s, t)=Q \frac{v^{4}}{u} \bar{D}_{4444}(v, Y) \\
& B_{1}(v, Y)=u v b_{1}(s, t)=Q\left(u-\frac{u}{v}-1\right) \frac{v^{4}}{u} \bar{D}_{4444}(v, Y) \\
& B_{2}(v, Y)=v b_{2}(s, t)=Q\left(1-u-\frac{u}{v}\right) \frac{v^{4}}{u^{2}} \bar{D}_{4444}(v, Y), \\
& B_{3}(v, Y)=u b_{3}(s, t)=Q\left(\frac{u}{v}-u-1\right) \frac{v^{3}}{u} \bar{D}_{4444}(v, Y) .
\end{aligned}
$$

The $\bar{D}_{4444}$ function has the following decomposition

$$
\begin{aligned}
\bar{D}_{4444} & =\frac{5 \pi^{2}}{108} \sum_{n, m=0}^{\infty} \frac{Y^{m}}{m !} \frac{v^{n}}{(n !)^{2}} \frac{\Gamma^{2}(n+4) \Gamma^{2}(n+m+4)}{\Gamma(8+2 n+m)} \\
& \times[-\log v+2 \psi(n+1)-2 \psi(n+4)-2 \psi(n+m+4)+2 \psi(8+2 n+m)] .
\end{aligned}
$$

We are now ready to analyze the contribution of the instantons to OPE of the lowest weight CPOs. Firstly we consider the short-distance expansion for the projection in the singlet. We find that the leading terms are given by

$$
\begin{aligned}
\left.\left\langle O^{I_{1}}\left(x_{1}\right) O^{I_{2}}\left(x_{2}\right) O^{I_{3}}\left(x_{3}\right) O^{I_{4}}\left(x_{4}\right)\right\rangle\right|_{\mathbf{1}} & =\frac{\pi^{2} Q \delta^{I_{1} I_{2}} \delta^{I_{3} I_{4}}}{x_{12}^{4} x_{34}^{4}}\left[-\frac{1}{84}(1+Y) v^{2} \log v\right. \\
& \left.-v^{2}\left(\frac{451}{17640}+\frac{139}{4410} Y\right)\right]
\end{aligned}
$$

This clearly shows that the leading contribution to the OPE comes from a scalar operator of approximate dimension 4. Our experience at strong coupling teaches us that the only such operator is the double-trace operator $O_{1}$ discussed in Section 3. On the other hand, due to the absence of $v \log v$ and $v Y^{2} \log v$-terms, the contribution of the Konishi fields $\mathcal{K}$ and $\mathcal{K}_{1}$, and of the operator $\Xi_{\mu \nu}$ are absent. We conclude that the Konishi multiplet as well as the multiplet built on $\Xi_{\mu \nu}$ receive only perturbative but not instanton corrections. ${ }^{8}$ Furthermore, we observe in (52) the absence of the contribution of the stress tensor, in agreement with the known non-renormalization theorem for this operator.

For the projection on $\mathbf{2 0}$ the leading contribution reads as

$$
\begin{aligned}
\left.\left\langle O^{I_{1}}\left(x_{1}\right) O^{I_{2}}\left(x_{2}\right) O^{I_{3}}\left(x_{3}\right) O^{I_{4}}\left(x_{4}\right)\right\rangle\right|_{\mathbf{2 0}} & =\frac{\pi^{2} Q C_{\mathcal{J}_{20}}^{I_{1} I_{2}} C_{\mathcal{J}_{20}}^{I_{3} I_{4}}}{x_{12}^{4} x_{34}^{4}}\left[-\frac{5}{252} v^{2} Y^{2} \log v\right. \\
& \left.-\frac{451}{10584} v^{2}\left(Y^{2}-v\right)\right]
\end{aligned}
$$

and comes from a second rank tensor of the canonical dimension 6. The contribution from the Konishi multiplet is again absent. Recall that at strong coupling we have found

\footnotetext{
${ }^{8}$ The absence of the instanton corrections to the Konishi multiplet was already noted in [35].
} 
that the first operators receiving anomalous dimension are scalar and tensor operators of approximate dimension 6 which we therefore identify with the operators appearing in (53).

These two examples at hand, i.e. projection in the singlet and in $\mathbf{2 0}$, allow us to make a general observation: the (double-trace) operators receiving finite anomalous dimensions at strong coupling also receive instanton contributions. The instanton contribution to the (single-trace) operators with infinite anomalous dimensions at strong coupling is absent.

Let us examine the other irreps. One gets the following leading behavior for 84:

$$
\left.\left\langle O^{I_{1}}\left(x_{1}\right) O^{I_{2}}\left(x_{2}\right) O^{I_{3}}\left(x_{3}\right) O^{I_{4}}\left(x_{4}\right)\right\rangle\right|_{\mathbf{8 4}}=\frac{\pi^{2} Q C_{\mathcal{J}_{84}}^{I_{1} I_{2}} C_{\mathcal{J}_{84}}^{I_{3} I_{4}}}{x_{12}^{4} x_{34}^{4}}\left[-\frac{1}{28} v^{3} \log v-\frac{451}{5880} v^{3}\right] ;
$$

for 105:

$$
\left.\left\langle O^{I_{1}}\left(x_{1}\right) O^{I_{2}}\left(x_{2}\right) O^{I_{3}}\left(x_{3}\right) O^{I_{4}}\left(x_{4}\right)\right\rangle\right|_{105}=\frac{\pi^{2} Q C_{\mathcal{J}_{105} I_{2}}^{I_{10} C_{\mathcal{J}_{105} I_{4}}^{I_{2}}}}{x_{12}^{4} x_{34}^{4}}\left[-\frac{1}{84} v^{4} \log v-\frac{451}{17640} v^{4}\right]
$$

for 15:

$$
\left.\left\langle O^{I_{1}}\left(x_{1}\right) O^{I_{2}}\left(x_{2}\right) O^{I_{3}}\left(x_{3}\right) O^{I_{4}}\left(x_{4}\right)\right\rangle\right|_{\mathbf{1 5}}=\frac{\pi^{2} Q C_{\mathcal{J}_{15}}^{I_{1} I_{2}} C_{\mathcal{J}_{15}}^{I_{3} I_{4}}}{x_{12}^{4} x_{34}^{4}}\left[-\frac{1}{21} v^{2} Y \log v-\frac{451}{4410} v^{2} Y\right]
$$

for 175:

$$
\left.\left\langle O^{I_{1}}\left(x_{1}\right) O^{I_{2}}\left(x_{2}\right) O^{I_{3}}\left(x_{3}\right) O^{I_{4}}\left(x_{4}\right)\right\rangle\right|_{\mathbf{1 7 5}}=\frac{\pi^{2} Q C_{\mathcal{J}_{175}}^{I_{1} I_{2}} C_{\mathcal{J}_{175} I_{4}}^{I_{1}}}{x_{12}^{4} x_{34}^{4}}\left[-\frac{1}{84} v^{3} Y \log v-\frac{451}{17640} v^{3} Y\right]
$$

Comparison of these formulae with the analogous strong coupling results confirms the above observation. Moreover, we see that the instanton contributions do not spoil the non-renormalization property of certain towers of double-trace operators found in [15]. Indeed, the scalar operator $O_{\mathbf{2 0}}$ in $\mathbf{2 0}$, all the rank $2 k$ tensors of dimension $4+2 k$ in $\mathbf{8 4}$ and of dimension $4+2 k, 6+2 k$ in $\mathbf{1 0 5}$, and all the rank $2 k+1$ tensors of dimension $5+2 k$ in 175 are non-renormalized in the instanton background.

The absence in the instanton OPE of corrections to the operators in the Konishi multiplet can be easily understood at follows. Instanton corrections to the normalization constant $C_{O O \mathcal{K}}$ and to the anomalous dimension of $\mathcal{K}$ are encoded into the 3 - and 2-point functions of $\mathcal{K}$ in the instanton background. According to the prescription of [38]-[41], to calculate the correlation functions of composite operators in the instanton background we replace the latter by their instanton background expressions; the resulting correlation function is then non-zero only if it contains all 16 fermionic zero modes. This is needed 
in order to saturate the fermionic integration measure. It is then easy to see that the following 3-point function

$$
\langle O O \mathcal{K}\rangle \sim\left\langle\operatorname{tr}\left(\phi^{\left(i_{1}\right.} \phi^{\left.i_{2}\right)}\right) \operatorname{tr}\left(\phi^{\left(i_{1}\right.} \phi^{\left.i_{2}\right)}\right) \operatorname{tr}\left(\phi^{k} \phi^{k}\right)\right\rangle
$$

contains only 12 fermionic zero modes and therefore it should be zero in the instanton background. Such arguments can be generalized to the whole Konishi multiplet. Indeed, as was shown in [43], the number of the fermion zero modes for an operators $O^{(q)}$ from the Konishi multiplet is $4-|q|$, where $q$ is a $U(1)_{Y}$ charge. The 3-point function of $O^{(q)}$ with two lowest-weight CPOs provides only $12-|q|$ zero modes and, therefore, vanishes.

Application of the same arguments to the double-trace operator $\mathcal{O}_{\mathbf{2 0}}$ shows that it can, in principle, receive instanton corrections. However, our explicit OPE calculation shows that it is not the case. This means that the particular dynamics that keeps $\mathcal{O}_{20}$ non-renormalized is not affected by instantons.

Concerning instanton corrections to the other multiplets we found, their existence is in agreement with the general considerations of [43]. An operator $O^{(q)}$ from a generic long multiplet provides $8-|q|$ zero modes, so that the 2- and 3-point correlation functions involving $O^{(q)}$ are non-zero only if $q=0$. Thus, in our OPE the leading operators that receive instanton corrections and survive at strong coupling with finite anomalous dimensions, are either primary operators (or conformal descendants of the primary operators), $O_{p}$ generating long multiplets or have the form $Q^{k} \bar{Q}^{k} O_{p}$.

Coming back to the singlet projection we now compute the instanton contribution $\Delta^{(i)}$ to the anomalous dimension of $O_{1}$. If we assume that the small parameter at hand is $\sim N^{1 / 2} / N^{4}$, then

$$
\frac{C_{O O O_{1}}^{2}}{2 C_{O_{1}}} \Delta_{1}^{(i)}=-\frac{\pi^{2} Q}{84}
$$

Substituting for $\frac{C_{O O O_{1}}}{C_{O_{1}}}$ its free-field value $1 / 10$ we get the anomalous dimension

$$
\Delta_{1}^{(i)}=-\frac{5 \pi^{2} Q}{21}
$$

By using the results of [15] we have also checked that the same ratio occurs for the leading operators in 84, 105 and 175 indicating thereby a universal behavior

$$
\frac{\Delta_{1}^{(i)}}{\Delta_{\mathbf{1}}^{(s)}}=\frac{\Delta_{\mathbf{1 5}}^{(i)}}{\Delta_{\mathbf{1 5}}^{(s)}}=\frac{\Delta_{\mathbf{8 4}}^{(i)}}{\Delta_{\mathbf{8 4}}^{(s)}}=\frac{\Delta_{\mathbf{1 0 5}}^{(i)}}{\Delta_{\mathbf{1 0 5}}^{(s)}}=\frac{\Delta_{\mathbf{1 7 5}}^{(i)}}{\Delta_{\mathbf{1 7 5}}^{(s)}}=\frac{5 \pi^{2}}{336} Q N^{2} .
$$




\section{Concluding remarks}

In this work we have extended the OPE analysis of the lowest weight CPOs initiated in [15], to include 2-loop and instanton contributions. At the perturbative level, we found that it is not sufficient to simply deform the free-field operator algebra by allowing for anomalous dimensions and corrections to the coupling in order to account for the 2-loop corrections. What is required is a splitting of various free-field operators into operators belonging to distinct supermultiplets which behave in a different way under the RG-flow. We have explicitly demonstrated this splitting in the case of the stress tensor and the $R$-current of the theory. Our results are consistent with earlier calculations by Anselmi in $[44,46]$. Furthermore, we argued that a similar splitting occurs also for free-field theory double-trace operators, e.g., they split into supermultiplets which behave in perturbation theory either like the Konishi multiplet or acquire anomalous dimensions $\sim \tilde{\lambda} / N^{2}$. The latter multiplets are the ones which survive at strong coupling and get non-zero anomalous dimensions. The above splitting seems to be necessary in order to explain the fact that while at any order in perturbation theory we expect a mixing of many operators with the same free-field dimension, at strong coupling only one of the operators is present while all the others decouple. Nevertheless, an explicit calculation of the the 2-loop anomalous dimension of the split operators would require the knowledge of 4-point functions including operators other than the lowest weight CPOs, e.g., the 4-point function of Konishi scalars. We believe that this is an interesting project.

We also found that the instantons give contributions only to operators which acquire non-zero and finite anomalous dimensions at strong coupling. In particular, instantons do not contribute neither to protected nor to operators in the Konishi multiplet. This is consistent with general arguments given in [43] concerning the vanishing of the three-point functions of operators with non-zero $U(1)_{Y}$-charge in the instanton background.

The fact that instantons contribute only to operators which correspond to "twoparticle" modes of classical supergravity, points to an interesting connection between the latter modes and D-particles modes which is worth further study. Intuitively, the corrections to the anomalous dimensions of the supergravity modes come from a corresponding change of their energy in the presence of $D$-particles. On the other hand, it can be seen that the universal behavior (57) for the leading operators in the OPE is a consequence of the fact that the correlation function of CPOs is defined (up to the free-field contribution) by a unique function $\mathcal{F}(v, Y)$. If one projects in a given irrep $\mathcal{J}$, one gets

$$
\left.\left\langle O^{I_{1}}\left(x_{1}\right) O^{I_{2}}\left(x_{2}\right) O^{I_{3}}\left(x_{3}\right) O^{I_{4}}\left(x_{4}\right)\right\rangle\right|_{\mathcal{J}}=\frac{C_{\mathcal{J}}^{I_{1} I_{2}} C_{\mathcal{J}}^{I_{3} I_{4}}}{x_{12}^{4} x_{34}^{4}}\left[h_{\mathcal{J}}(v, Y)+f_{\mathcal{J}}(v, Y) \mathcal{F}(v, Y)\right],
$$


where $h_{\mathcal{J}}(v, Y)$ is a free-field contribution, and $f_{\mathcal{J}}(v, Y)$ is some function depending on the irrep we consider. Now we see that if the strong coupling and instanton 4-point functions are described by $\mathcal{F}^{(s)}(v, Y)$ and $\mathcal{F}^{(i)}(v, Y)$ respectively, then after subtracting the free-field theory contribution, the ratio of the strong to the instanton contributions will be one and the same for all irreps since the function $f_{\mathcal{J}}(v, Y)$ does not depend on the regime we consider and, therefore, cancels out. What is more important is that the short-distance expansion of the both functions $f_{\mathcal{J}}(v, Y) \mathcal{F}^{(i, s)}(v, Y)$ starts as $f_{\mathcal{J}}(v, Y) \mathcal{F}^{(i, s)}(v, Y)=v^{\frac{\Delta-l}{2}} G_{l}^{(i, s)}(Y) \log v+\ldots$, where the function $G_{l}^{i, s}(Y)$ may be represented as $G_{l}^{(i, s)}(Y)=a_{\mathcal{J}}(Y) f^{(i, s)}(Y)=a Y^{l}+\ldots$ and it encodes the leading contribution of the rank- $l$ tensors of a canonical dimension $\Delta$. Therefore, the equalities (57) stem from the fact that the ratio of the instantons to strong-coupling corrections $\frac{G_{l}^{(i)}(Y)}{G_{l}^{(s)}(Y)}$ does not depend on an irrep we consider.

Finally, we wish to comment on the fact that we have confirmed both in perturbation theory and in the instanton background the non-renormalization of various operators, most importantly the scalar double-trace operator in the $\mathbf{2 0}$ with dimension 4 . The dimension of the latter operator is not protected by unitarity constraints and therefore it is subject to a genuine dynamical protection. Being a double-trace operator, it is difficult to find the corresponding supergravity mode. Furthermore, since it is a marginal operator we may use it to deform the $\mathcal{N}=4 \mathrm{SYM}_{4}$ action preserving the conformal invariance to leading order in the deformation. It would be of great interest to find out if this operator is exactly marginal as in this case it defines a particular RG flow (fixed line), that might lead to a new non-trivial $\mathcal{N}=0 \mathrm{CFT}$.

Acknowledgements We would like to thank A. Tseytlin for useful comments on the manuscript. G. A. and T. P. are grateful to S. Ferrara and P. Fre' for useful discussions. G. A. would like to acknowledge helpful conversations with S. Theisen. The work of G. A. was supported by the DFG and by the European Commission RTN programme HPRN-CT-2000-00131 in which G. A. is associated to U. Bonn, and in part by RFBI grant N99-01-00166. The work of S.F. was supported by the U.S. Department of Energy under grant No. DE-FG02-91ER-40690 and in part by RFBI grant N99-01-00190. T. P. was supported by the E.U. under the program RTN1-1999-00116. 


\section{References}

[1] J. Maldacena, "The large $N$ limit of superconformal field theories and supergravity", Adv. Theor. Math. Phys. 2 (1998) 231.

[2] G.G. Gubser, I.R. Klebanov and A.M. Polyakov, "Gauge theory correlators from noncritical string theory", Phys.Lett. B428 (1998) 105, hep-th/9802109.

[3] E. Witten, "Anti-de Sitter space and holography", Adv.Theor.Math.Phys. 2 (1998) 253, hep-th/9802150.

[4] T. Banks and M. B. Green, "Non-perturbative effects in $\operatorname{AdS}(5)$ x S**5 string theory and d = 4 SUSY Yang-Mills," JHEP 9805 (1998) 002, hep-th/9804170.

[5] S. Lee, S. Minwalla, M. Rangamani and N. Seiberg, "Three-point functions of chiral operators in $\mathrm{D}=4, \mathrm{~N}=4 \mathrm{SYM}$ at large N," Adv. Theor. Math. Phys. 2 (1998) 697, hepth/9806074.

[6] E. D'Hoker, D. Z. Freedman and W. Skiba, "Field theory tests for correlators in the AdS/CFT correspondence," Phys. Rev. D59 (1999) 045008, hep-th/9807098.

[7] W. Skiba, "Correlators of short multi-trace operators in N = 4 supersymmetric Yang-Mills," Phys. Rev. D60 (1999) 105038, hep-th/9907088.

[8] F. Gonzalez-Rey, B. Kulik and I. Y. Park, "Non-renormalization of two point and three point correlators of $\mathrm{N}=4 \mathrm{SYM}$ in $\mathrm{N}=1$ superspace," Phys. Lett. B455 (1999) 164, hep-th/9903094.

[9] A. C. Petkou and K. Skenderis, "A non-renormalization theorem for conformal anomalies", Nucl Phys. B561 (1999) 100, hep-th/9906030.

[10] S. Penati, A. Santambrogio and D. Zanon, "Two-point functions of chiral operators in N = 4 SYM at order g**4," JHEP 9912 (1999) 006, hep-th/9910197.

[11] S. Penati, A. Santambrogio and D. Zanon, "More on correlators and contact terms in N = 4 SYM at order g**$^{* *}, "$ hep-th/0005223.

[12] P. S. Howe, E. Sokatchev and P. C. West, "3-point functions in N = 4 Yang-Mills," Phys. Lett. B444 (1998) 341, hep-th/9808162.

[13] G. Arutyunov and S. Frolov, "Scalar Quartic Couplings in Type IIB Supergravity on $A d S_{5} \times$ $S^{5 ",}$ Nucl.Phys. B579 (2000) 117, hep-th/9912210.

[14] G. Arutyunov and S. Frolov, "Four-point Functions of Lowest Weight CPOs in $\mathcal{N}=4$ $\mathrm{SYM}_{4}$ in Supergravity Approximation", Phys. Rev. D62 (2000) 064016, hep-th/0002170. 
[15] G. Arutyunov, S. Frolov and A. C. Petkou, "Operator Product Expansion of the Lowest Weight CPOs in $\mathcal{N}=4 \mathrm{SYM}_{4}$ at Strong Coupling", Nucl.Phys. B586 (2000) 547, hepth/0005182.

[16] H. Liu and A.A. Tseytlin, "On four-point functions in the CFT/AdS Correspondence", Phys.Rev. D59 (1999) 086002, hep-th/9807097.

[17] D. Freedman, S.D. Mathur, A. Matusis and L. Rastelli, "Comments on 4-point functions in the CFT/AdS correspondence", Phys.Lett. B452 (1999) 61, hep-th/9808006.

[18] G. Chalmers and K. Schalm, "The large $N_{c}$ limit of four-point functions in $N=4$ super Yang-Mills theory from Anti-de Sitter supergravity",Nucl.Phys. B554 (1999) 215, hepth/9810051.

[19] E. D'Hoker and D. Freedman, "Gauge boson exchange in $A d S_{d+1}$ ", Nucl.Phys. B544 (1999) 612, hep-th/9809179.

[20] J.H. Brodie and M. Gutperle, "String corrections to 4-point functions in the AdS/CFT correspondence", Phys.Lett. B445 (1999) 296, hep-th/9809067.

[21] H. Liu, "Scattering in Anti-de Sitter space and operator product expansion", Phys. Rev. D60 (1999) 106005, hep-th/9811152.

[22] E. D'Hoker and D. Freedman, "General Scalar Exchange in $\mathrm{AdS}_{d}+1$ ", Nucl.Phys. B550 (1999) 612, hep-th/9811257.

[23] E. D'Hoker, D. Freedman, S. Mathur, A. Matusis and L. Rastelli, "Graviton exchange and complete 4-point functions in the AdS/CFT correspondence", Nucl.Phys. B562 (1999) 353, hep-th/9903196.

[24] E. D'Hoker, D. Freedman and L. Rastelli, "AdS/CFT 4-point functions: How to succeed at z-integrals without really trying", Nucl.Phys. B562 (1999) 395, hep-th/9905049.

[25] Sanjay, "On direct and crossed channel asymptotics of four-point functions in AdS/CFT correspondence", Mod.Phys.Lett. A14 (1999) 1413, hep-th/9906099.

[26] E. D'Hoker, S.D. Mathur, A. Matusis and L. Rastelli, "The Operator Product Expansion of $\mathcal{N}=4 \mathrm{SYM}$ and the 4-point Functions of Supergravity", hep-th/9911222.

[27] L. Hoffmann, A.C. Petkou and W. Rühl, "A note on the analyticity of AdS scalar exchange graphs in the crossed channel", Phys. Lett. B478 (2000) 320, hep-th/0002025.

[28] C.P. Herzog, "OPEs and 4-point functions in AdS/CFT correspondence", hep-th/0002039. 
[29] L. Hoffmann, A.C. Petkou and W. Rühl, "Aspects of the conformal Operator Product Expansion in AdS/CFT correspondence", hep-th/0002154.

[30] L. Hoffmann, L. Mesref and W. Ruhl, "AdS box graphs, unitarity and operator product expansions," hep-th/0006165.

[31] S. Ferrara and A. Zaffaroni, "Superconformal Field Theories, Multiplet Shortening and the $\mathrm{AdS}_{5} / \mathrm{SCFT}_{4}$ Correspondence", hep-th/9908163.

[32] F. Gonzalez-Rey, I. Park and K. Schalm, "A note on four-point functions of conformal operators in N=4 Super-Yang Mills", Phys. Lett. B448 (1999) 37, hep-th/9811155.

[33] B. Eden, P.S. Howe, C. Schubert, E. Sokatchev and P.C. West, "Four-point functions in N=4 supersymmetric Yang-Mills theory at two loops", Nucl.Phys. B557 (1999) 355, hepth/9811172.

[34] B. Eden, P.S. Howe, C. Schubert, E. Sokatchev and P.C. West, "Simplifications of fourpoint functions in $\mathcal{N}=4$ supersymmetric Yang-Mills theory at two loops", Phys. Lett. B466 (1999) 20, hep-th/9906051.

[35] M. Bianchi, S. Kovacs, G. Rossi and Y.S. Stanev, "On logarithmic behaviour in $\mathcal{N}=4$ SYM theory", JHEP 9908 (1999) 020, hep-th/9906188.

[36] B. Eden, C. Schubert and E. Sokatchev, "Three-loop four-point correlator in $\mathcal{N}=4$ SYM", Phys.Lett. B482 (2000) 309, hep-th/0003096.

[37] M. Bianchi, S. Kovacs, G. Rossi and Y.S. Stanev, "Anomalous dimensions in $\mathcal{N}=4$ SYM at order $g^{4} "$, Nucl.Phys. B584 (2000) 216, hep-th/0003203.

[38] M. Bianchi, M. Green, S. Kovacs and G. Rossi, "Instantons in supersymmetric Yang-Mills and D-instantons in IIB superstrings theory", JHEP 9808 (1998) 013, hep-th/9807033.

[39] N. Dorey, V. Khoze, M. Mattis and S. Vandoren, "Yang-Mills Instantons in the Large-N Limit and the AdS/CFT Correspondence", Phys.Lett. B442 (1998) 145, hep-th/9808157.

[40] N. Dorey, T. Hollowood, V. Khoze, M. Mattis and S. Vandoren, "Multi-Instantons and Maldacena's Conjecture", JHEP 9906 (1999) 023, hep-th/9810243.

[41] N. Dorey, T. Hollowood, V. Khoze, M. Mattis and S. Vandoren, "Multi-Instanton Calculus and the AdS/CFT Correspondence in N=4 Superconformal Field Theory", Nucl.Phys. B552 (1999) 88, hep-th/9901128.

[42] B. Eden, A. Petkou, C. Schubert and E. Sokatchev, "Partial non-renormalization of the stress-tensor four-point function in N = 4 SYM and AdS/CFT," hep-th/0009106. 
[43] K. Intriligator and W. Skiba, "Bonus symmetry and the Operator Product Expansion of $\mathcal{N}=4$ Super-Yang-Mills", hep-th/9905020.

[44] D. Anselmi, "The $N=4$ quantum conformal algebra", Nucl. Phys. B541 (1999) 369, hep-th/9809195.

[45] H. Osborn and A. C. Petkou, "Implications of conformal invariance in field theories for general dimensions", Ann. Phys. 231 (1994) 311, hep-th/9307010.

[46] D. Anselmi, "Quantum conformal algebras and closed conformal field theory", Nucl. Phys. B554 (1999) 415, hep-th/9811149. 